\title{
Androgensuppression lohnt sich auch bei lokal begrenztem Prostatakarzinom
}

Die kombinierte Therapie mit Bestrahlung und Androgensuppression (AS) ist bei lokal fortgeschrittenem Prostatakarzinom (PCA) etabliert. Ihr Stellenwert bei lokal begrenztem Prostatakarzinom mit mittlerem bis hohem Risiko (5-Jahres-Rezidivrisiko $30 \%$ ) war bisher unklar. Nun liegen erste Daten vor.

n der EORTC-Studie 22991 wurde untersucht, ob sich das Überleben von PCA-Patienten im Stadium cT1b-c (PSAWert $\geq 10 \mathrm{ng} / \mathrm{ml}$ oder Gleason-Score $\geq 7$ ) oder cT2a (PSA-Wert $\leq 50 \mathrm{ng} / \mathrm{ml}$, nodal negativ und nicht metastasiert) ohne biochemisches Rezidiv durch eine adjuvante AS Monate verbessert. Die 819 Patienten

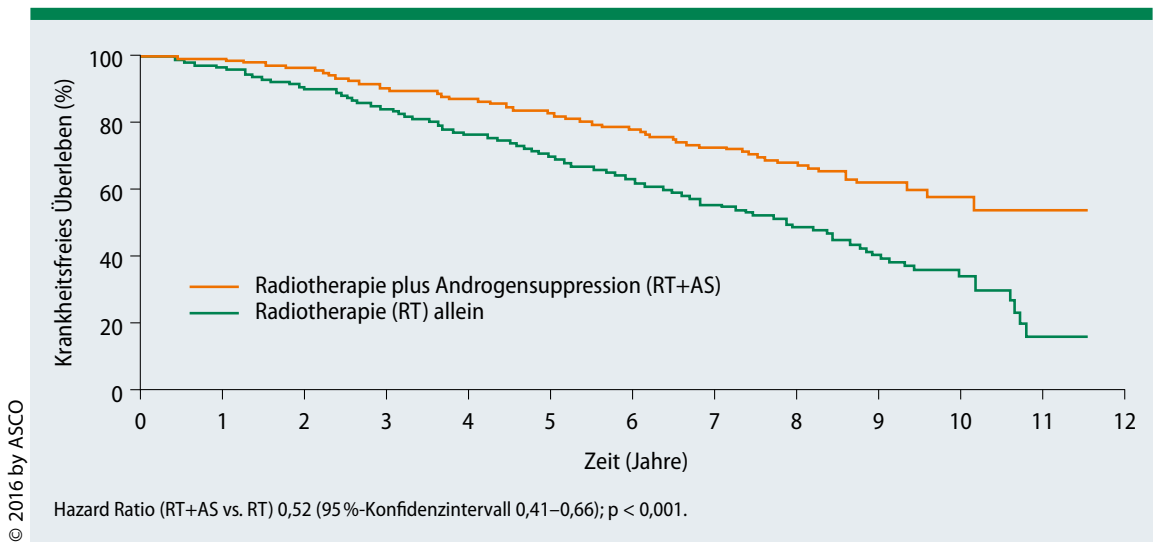

Abb. 1: Überleben ohne biochemisches Rezidiv in der Intent-to-treat-Population der Studie: Die zusätzliche Androgensuppression zahlt sich aus.

\section{PSA-Anstieg nach Prostatektomie: längeres PFS durch vorübergehende Androgensuppression}

Wie behandelt man einen steigenden PSA-Wert nach radikaler Prostatektomie am besten? Welchen Nutzen bietet eine Kurzzeit-Androgensuppression (AS)? Dieser Frage gingen franösische Urologen nach.

K ommt es nach radikaler Prostatektomie zu einem PSA-Anstieg ohne Anzeichen einer klinischen oder radiologischen Erkrankung, ist die Standardtherapie eine Salvage-Bestrahlung. Allerdings kommt es bei mehr als der Hälfte der Patienten binnen 5 Jahren trotzdem zum biochemischen Rezidiv. In der Phase-IIIStudie GETUG-AFU 16 erhielten daher 743 Männer mit Adenokarzinom der Pro- stata und PSA-Anstieg von 0,2 auf $<2,0 \mu \mathrm{g} / \mathrm{l}$ nach radikaler Prostatektomie randomisiert eine Standard-Salvage-Radiotherapie (RT) mit 66 Gy in 33 Fraktionen) oder eine RT in Kombination mit einer 6-monatigen AS (RT+AS). Nach median 63 Monaten hatten die Patienten im RT+AS-Arm signifikant häufiger keine biochemische und auch keine klinische Progression (80 vs. $62 \%$; Hazard Ratio [HR] 0,50; $\mathrm{p}<0,0001)$. Unter RT+AS waren $18 \%$ der Patienten progredient, unter RT allein $33 \%$, wobei zudem die Zeit bis zur Progression länger war (median 32 vs. 22 Monate, $\mathrm{p}=0,0001$ ).

Auf das Gesamtüberleben hatte die Androgensuppression allerdings keinen signifikanten Einfluss: weder auf das (medianes Alter 70 Jahre) wurden randomisiert entweder nur bestrahlt (RT) oder sie wurden bestrahlt und erhielten zusätzlich eine AS-Therapie für 6 Monate (RT+AS). 74,8\% der Patienten hatten ein mittleres, 24,8\% ein hohes Risiko.

Nach einem Follow-up von median 7,2 Jahren war das Überleben ohne biochemisches Rezidiv und klinische Progression in der RT+AS-Gruppe signifikant besser (Abb. 1). Nur 28,8\% der Patienten in der RT+AS-Gruppe entwickelten ein biochemisches Rezidiv (49,1\% in der RTGruppe). Auch das 5-Jahres-Überleben ohne biochemisches Rezidiv war besser (Hazard Ratio [HR] 0,52; p < 0,001). Lokalrezidive und Fernmetastasen waren in der RT+AS-Gruppe nach 5 Jahren seltener $(2,1$ vs. $6,6 \%$; HR 0,37; $\mathrm{p}=0,001$ bzw. 4,4 vs. $7,6 \%$; $\mathrm{p}=0,05)$.

Fazit: Eine adjuvante AS über 6 Monate verbessert das biochemisch und klinisch progressionsfreie Überleben bei Patienten, die wegen eines Prostatakarzinoms mit mittlerem oder hohem Risiko bestrahlt werden, signifikant JudithNeumaier

Bolla M et al. Short Androgen Suppression and Radiation Dose Escalation for Intermediate- and High-Risk Localized Prostate Cancer: Results of EORTC Trial 22991. J Clin Oncol. 2016;34(15): 1748-56.

mediane Gesamtüberleben (58 vs. 56 Monate) noch auf das 5-Jahres-Gesamtüberleben (96 vs. $95 \%$; HR 0,7; $\mathrm{p}=0,18$ ).

Fazit: Männer mit PSA-Anstieg nach radikaler Prostatektomie profitieren von kurzzeitiger AS zusätzlich zur SalvageRadiotherapie. Daher sollte diese Therapie in Betracht gezogen werden, vor allem bei Männern mit hohem Risiko. Durch Verlängerung des progressionsfreien Überlebens kann man aggressivere Therapien wie eine langfristige AS und die damit einhergehenden Beeinträchtigungen hinauszögern.

Judith Neumaier

Carrie $\mathrm{C}$ et al. Salvage radiotherapy with or without short-term hormone therapy for rising prostate-specifi c antigen concentration after radical prostatectomy (GETUG-AFU 16): a randomised, multicentre, open-label phase 3 trial. Lancet Oncol. 2016;17(6):747-56. 